\title{
On the Equivalence of the Two Theories of the Single Phase Induction Motor
}

\author{
BY V. KARAPETOFF \\ Professor of Electrical Engineering, Cornell University.
}

\begin{abstract}
It is shown that the two equivalent diagrams of a single-phase induction motor, viz., the one deduced from the revolving-field theory and that from the cross-flux theory, can be converted into each other. In other words, the two theories iead to the same performance of a motor with given design constants. It is also shown that many other equivalent diagrams are possible and that one of these contains a variable impedance depending upon the slip in one parallel branch only, the other parts of the circuit being constant.
\end{abstract}

$\mathrm{T}$ HERE are two theories of the single-phase induction motor that are in general use among engineers, viz.:

1. The rotating-field theory, according to which the single-phase motor is replaced by two polyphase motors in torque opposition, with the stator windings in series and with separate rotors. This theory leads to the equivalent diagram shown in Fig. 1.

2. The cross-field theory, according to which there are two separate air-gap fields, one in space phase

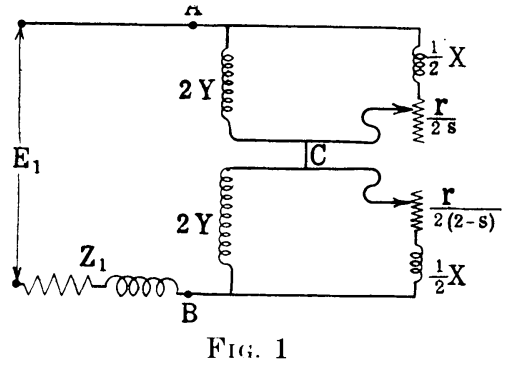

and the other in space quadrature with the stator winding. This theory leads to the equivalent diagram shown in Fig. 2.

These two theories are considered in detail in E. Arnold's Wechselstromtechnik, Vol. V., Part 1, (1909) Chaps. 7 and 8 , and the equivalent diagrams are proved there. Each theory leads to a circle diagram for the locus of the primary current, and on p. 15 Arnold shows that three points on the two circles coincide. From this fact he concludes with sufficient reason that the two theories lead to an identical performance for a given motor, and that if one of the two theories is correct, the other must be correct.

The process of finding the position of the center and the radius of the circle according to either theory is quite tedious, and the resulting graphical construction rather involved. On the other hand, the equivalent diagrams shown in Figs. 1 and 2 can be used analytically for the predetermination of performance or for any other investigation of the motor, without representing them graphically. It is of interest therefore to show directly the equivalence of the two diagrams of connections and to deduce a method of transforming one into the other.

In both figures, $E_{1}$ is the line voltage and $Z_{1}$ is the primary impedance consisting of the resistance of the primary winding and of its leakage reactance. Since $Z_{1}$ is taken into account in the same manner in both diagrams we are not further concerned with it in our comparison of the two diagrams. $Y$ is the exciting admittance of the main magnetic circuit and is shown in the usual manner in Fig. 2. In Fig. 1 this admittance is shown separately as $2 Y$ for each of the two component polyphase motors. Each fictitious component motor has only one-half as many turns as the actual motor and for this reason the exciting admittance has to be doubled (Arnold, ibid., p. 152.)

The meaning of the other symbols in the figures is as follows: $X=j x_{2}$ is the secondary leakage reactance and is used in the analytical part of this treatment as an operator in complex notation. The resistance $r$ is that of the rotor, considering it as a single-phase circuit reduced to the primary number of turns. It is known from the general theory of the po yphase induction motor that in the equivalent electric diagram the load and the secondary resistance together are replaced by the resistance $r_{/}^{/} s$, where $s$ is the slip of the machine (see, for example, the author's "Electric Circuit," p. 123). One of the component machines in Fig. 1 runs at a slip $s$, the other at the slip $2-s$. Moreover, only one-half of the resistance of the actual

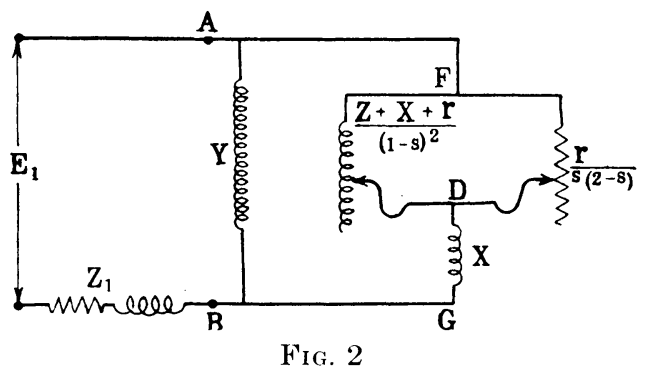

single-phase machine must be assigned to each polyphase machine. This explains the meaning of the resistances $\begin{array}{ccc}1 & r \\ 2 & s\end{array}$ and $\frac{1}{2}-\frac{r}{(2-s)} \cdot$ For the same reason $-\frac{1}{2} X$ is assigned to each polyphase motor. The same quantities enter in Fig. 2 in a different form resulting from the crossfield theory. The impedance $Z$ in this diagram is that of the main exciting circuit, so that $Z=\frac{1}{Y}$

For the sake of abbreviation we shall also introduce the following notation:

$$
\begin{aligned}
& Z^{\prime}=\frac{1}{Y^{\prime}}=\frac{1}{2} X+\frac{1}{2} \frac{r}{s} \\
& Z^{\prime \prime}=\frac{1}{Y^{\prime}}=\frac{1}{2} X+\frac{1}{2} \frac{r}{(2-s)}
\end{aligned}
$$


The total impedance between the points $A$ and $C$ in Fig. 1 is equal to $\left(2 Y+Y^{\prime}\right)^{-1}$, and that between $C$ and $B$ is $\left(2 Y+Y^{\prime \prime}\right)^{-1}$. Hence, the total equivalent impedance between the points $A$ and $B$ is

$$
\begin{aligned}
Z_{\mathrm{AB}}=\frac{1}{2 Y+Y^{\prime}}+\frac{1}{2 Y+Y^{\prime \prime}} \\
=\frac{4 Y+Y^{\prime}+Y^{\prime \prime}}{\left(2 Y+Y^{\prime}\right)\left(2 Y+Y^{\nu}\right)}
\end{aligned}
$$

If the diagrams in Figs. 1 and 2 are equivalent, then equation (3) must also give the value of the total impedance between the points $A$ and $B$ in Fig. 2 . To get the impedance between the points $F$ and $G$, the admittance $Y$ must be subtracted from $Z_{\mathrm{AB}}{ }^{-1}$. We then get

$$
Y_{\mathrm{FG}}=Y_{\mathrm{AB}}-Y=Z_{\mathrm{AB}}{ }^{-1}-Y
$$

or, using the value of $Z_{\mathrm{AB}}$ from equation (3), we obtain, after reduction

$$
Y_{\mathrm{FG}}=-\frac{Y Y^{\prime}+Y Y^{\prime \prime}+Y^{\prime} Y^{\prime \prime}}{4 Y+Y^{\prime}+Y^{\prime \prime}}
$$

The corresponding impedance is

$$
Z_{\mathrm{FG}}=Y_{\mathrm{FG}}{ }^{-1}=\frac{4 Y+Y^{\prime}+Y^{\prime \prime}}{Y Y^{\prime}+Y Y^{\prime \prime}+Y^{\prime} Y^{\prime \prime}}
$$

Multiplying the numerator and the denominator of this fraction by $Z Z^{\prime} Z^{\prime \prime}$ and remembering that the $Z$ 's are the reciprocals of the corresponding $Y$ 's, we get

$$
Z_{\mathrm{FG}}=\frac{4 Z^{\prime} Z^{\prime \prime}+Z Z^{\prime \prime}+Z Z^{\prime}}{Z^{\prime \prime}+Z^{\prime}+Z}
$$

In order to obtain the impedance between the points $D$ and $F$, the reactance $X$ has to be subtracted from the foregoing expression. We get then, after simple algebraic transformations,

$Z_{\mathrm{FD}}=Z_{\mathrm{FG}}-X=$

$$
\frac{\left(2 Z^{\prime}-X\right) Z^{\prime \prime}+\left(2 Z^{\prime \prime}-X\right) Z^{\prime}+Z\left(Z^{\prime}+Z^{\prime \prime}-X\right)}{Z^{\prime \prime}+Z^{\prime}+Z}
$$

With the help of equations (1) and (2), this is directly reduced to

$$
\begin{aligned}
Z_{\mathrm{FD}} & =\frac{Z^{\prime \prime} r / s+Z^{\prime} r /(2-s)+Z r / s(2-s)}{Z+X+r / s(2-s)} \\
& =r \frac{Z+X+r}{(Z+X) s(2-s)+r}
\end{aligned}
$$

The total admittance between the points $F$ and $D$ is

$Y_{\mathrm{FD}}=Z_{\mathrm{FD}}^{-1}=-\frac{1}{r} \frac{(Z+X) s(2-s)+r}{Z+X+r}$

By adding and subtracting $r$ in the parentheses of the first term of the numerator, this expression is transformed into

$$
Y_{\mathrm{FD}}=\frac{1}{r} \frac{(Z+X+r) s(2-s)+[r-r s(2-s)]}{Z+X+r}
$$

or, writing the denominator separately under each term,

$$
Y_{\mathrm{FD}}=\frac{s(2-s)}{r}+\frac{(1-s)^{2}}{Z+X+r}
$$

Expression (11) represents two admittances in parallel and means that the circuit between $F$ and $D$ consists of the resistance $\frac{r}{s(2-s)}$ in parallel with the impedance $\frac{(Z+X+r)}{(1-s)^{2}}$. This conclusion agrees with the values shown in Fig. 2, which have been induced independently, from the cross-field theory. The equivalence of the two theories is thus proved.

Equation (10) is shown below to lead also to the equivalent diagram in Fig. 3. This diagram has the

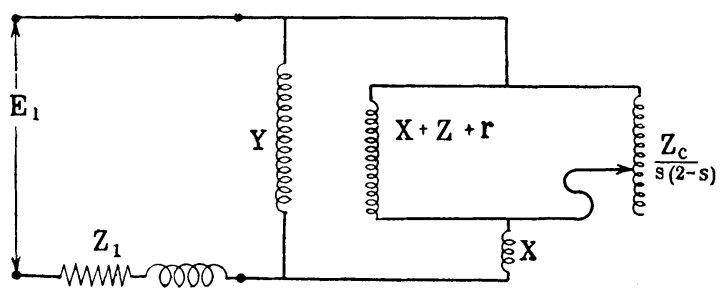

FIG. 3 .

advantage over Fig. 2 in that the impedance of one branch only is a function of $s$ and varies with the load. As a matter of fact, the total admittance $Y_{\mathrm{FD}}$ may be resolved into two or more admittances in parallel or impedances in series in an infinite variety of ways, so that there is an infinitely great number of equivalent diagrams of the kind shown in Figs. 2 and 3. All of them are characterized by the shunted exciting admittance $Y$ and by the series reactance $X$.

To prove that equation (10) leads to Fig. 3, we simply write the denominator separately under each term and thus obtain

$$
Y_{\mathrm{FD}}=\frac{(Z+X) s(2-s)}{r(Z+X+r)}+\frac{1}{Z+X+r}
$$

This shows that $Y_{\mathrm{FD}}$ consists of two impedances in parallel, namely $Z+X+r$, and $\frac{Z_{c}}{s(2-s)}$ where

$$
Z_{c}=r \frac{Z+X+r}{Z+X}
$$

The fraction by which $r$ is multiplied is a known complex quantity of the form $a+j b$, so that

$$
Z_{c}=r a+j r b
$$

and this value is used in Fig. 3.

Neglecting the core loss, the impedance $Z$ is a pure reactance, so that approximately we may put the foregoing ratio in the form

$$
1+\frac{r}{Z+X}=1-j \tan \phi
$$

where $\phi$ is a small known angle which is constant for a given motor and is determined by the constants $r, Z$, and $X$ of the machine. Hence, approximately

$$
Z_{c}=r-j r \tan \phi=\frac{r}{\cos \phi} \epsilon^{-j \phi}
$$

Two noteworthy papers on the single-phase induction motor, by Hellmund and by Lamme, as well as an extensive and stimulating discussion by others, will be found in the A. I. E. E. Trans., Vol. 37 (1918), pp. 539-702. 